\title{
Envejecimiento de la población: un reto para las políticas públicas del siglo XXI
}

María Teresa Bazo Royo *

Vivimos en lo que se denomina sociedades envejecidas. El término -ampliamente utilizado en las ciencias gerontológicas- hace referencia a los cambios en la distribución de edad de la población, en la que la proporción de personas ancianas en relación a las jóvenes está aumentando rápidamente. Implica cambios en el resto de las estructuras. En el caso de España las personas de sesenta y cinco y más años representaban a principios de siglo el 5,2\% de la población, en 1979 el 9,7\% $y$ en 1991 el 13,7\%. Para el año 2005, según datos del INE, se estima una proporción del $17,4 \%$. En un siglo la proporción se ha más que multiplicado por tres. El envejecimiento de la población es debido a la conjunción de dos factores: el descenso de la natalidad y el descenso de las tasas de mortalidad (sobre todo, las de mortalidad infantil) que es el fenómeno demográfico más importante del presente siglo.

Los cambios en los roles de las mujeres, debido en parte al aumento de los niveles de educación y en parte también a la mayor efectividad de los métodos anticonceptivos, contribuyen al descenso de las tasas de natalidad, que a su vez influyen en el aumento de la proporción de personas ancianas. Por otra parte, el aumento de la esperanza de vida al nacer, e incluso a la edad de jubilación, lleva al incremento del volumen de personas mayores. La prolongación de la esperanza de vida es resultado de las luchas contra las enfermedades infecciosas que causaban la muerte en épocas anteriores, así como de los avances médicos en el tratamiento de las enfermedades crónicas. La esperanza de vida, junto con la tasa de mortalidad infantil, es considerado el mejor índice sintético de la calidad de vida de una sociedad, pues refleja las condiciones económicas, culturales, y sociales de la misma Que las personas vivan más años es, por tanto, un logro social. Viven más y en mejor estado de salud que nunca. Las sociedades envejecidas son resultado del desarrollo social que viene acompañando en nuestras sociedades al desarrollo económico. Hoy existe lo que denominamos una «nueva vejez» (BAzO, 1992a).

La esperanza de vida al nacer de las mujeres en España es de más de ochenta y un años (WALKER y MALtBY, 1997: 14) y todavía a los sesenta años de edad es de veinticuatro años más (p. 13), siendo ésta la segunda más alta de la Unión Europea después de Francia, y por delante de la desarrollada Suecia. El grupo de edad que crece a mayor ritmo es el de las personas octogenarias. En España las personas de ochenta y más años representan el 2,9\% del total de la población ( $\mathrm{S}_{\mathrm{AL}}$. VAGE, 1995), lo que supone que algo más de un millón de españoles tienen ochenta y más años. Una característica esencial de la ancianidad es la feminización. En España por cada 100 mujeres de sesenta y cinco y más años hay 70 varones del mismo grupo de edad. Esa razón es más alta en los grupos jóvenes, y desciende a 45 varones por cien mujeres entre las personas de ochenta y cinco y más años. Las personas ancianas en su conjunto en España - como en otras sociedades desarrolladas - manifiestan de forma mayoritaria encontrarse bien de salud para realizar las actividades de la vida cotidiana. Alrededor del $80 \%$ de quienes viven en la comunidad declaran no necesitar ayuda. Puede decirse que son personas independientes (FER. NÁNDEZ-Mayoralas y RodrígueZ RodRígueZ, 1995; Béland y ZunZUNEGUI, 1995). Además, la mayor parte de las personas mayores manifiesta sentirse bien de salud (INSERSO, 1995a; De Miguel, 1994; Vallés y Cea d’Ancona, 1994; CIRES, 1993; Bazo, 1990, y Cruz y Cobo, 1990).

La mayor parte de las personas ancianas que están en buen estado de salud relativo se encuentran entre los grupos más 
jóvenes, entre las que Bernice NeUGARTEN (1974) denomina los «viejos-jóvenes» (young-old). Debe tenerse en cuenta de cara a la estimación de los niveles de salud de las personas ancianas que suelen manejarse datos sobre población mayor no institucionalizada. En la sociedad española, además, deben tenerse en cuenta dos hechos: que como en otras sociedades las personas ancianas en residencias tienden a ser más viejas y más enfermas y discapacitadas que el resto de personas ancianas. Otra cuestión es que, dada la escasez de recursos formales para atender las necesidades sociosanitarias de la población anciana que precisa cuidados, la gran mayoría de las personas discapacitadas o con enfermedades crónicas es cuidada en familia, y puede añadirse que, en la inmensa mayoría de los casos, sin ningún apoyo exterior (BAZO, 1998). La edad no es el mejor predictor del estado de salud para una determinada persona, pero es un buen predictor de las tasas de enfermedad para grupos de personas. Con la edad aumenta la probabilidad de sufrir enfermedades crónicas y discapacidad (COLLADO, Domín. GUEZ y DE Miguel, 1991). Existe también una morbilidad diferencial por género. Las mujeres, por las características de sus enfermedades específicas, sufren más discapacidad que los varones. Las enfermedades de los varones les llevan a morir Además, como ellas viven más que ellos, el aumento de edad implica una probabilidad mayor de incapacitación para llevar una vida independiente.

Aun así, ellas viven más a solas que ellos, en una relación de ocho a dos (son las que mejor se encuentran). Incluso en los grupos de ochenta y más años ellos viven a solas en el $7 \%$ de los casos, y ellas en el 23\% (INSERSO, 1995a: 44). El grupo de más edad es el que conlleva más riesgo de discapacidad, y la mayoría de las personas octogenarias son mujeres (79\% según el ratio de masculinidad) tendiendo a mantenerse en su hogar mientras pueden, aunque además son más pobres que los varones. Vivir a solas no significa sentirse solo, pero algunas personas ancianas experimentan un sentimiento intenso de soledad, lo que se asocia con percepciones más negativas de su salud y bienestar general (BAZO, 1990). Todo ello -es obvio - tiene que tener consecuencias en las políticas sanitarias y sociales. En las personas de edad es más probable sufrir enfermedades crónicas que agudas. No obstante, la enfermedad y la discapacidad no desembocan necesariamente en la minusvalía. El número e importancia de las minusvalías funcionales son una medida mejor del estado de salud que la presencia de ciertas enfermedades. Las personas suelen declararse enfermas cuando sus problemas de salud les impiden la realización de las actividades de la vida diaria, es decir, cuando les dificulta en mayor o menor grado su cuidado personal y la atención de su hogar y las tareas que eso conlleva.

En España, entre las personas de ochenta y más años que residen en la comunidad, excepto para bañarse o ducharse, al rededor de las tres cuartas partes como mínimo pueden realizar el resto de actividades sin necesidad de ayuda (INSERSO, 1995a: 56). Son las personas - generalmente entre las de más edad - que sufren las peores condiciones de salud física y mental, así como pérdidas sociales, las que requieren la atención mayor por parte de las políticas y servicios sociales. Algunas se encuentran en residencias, pero la mayoría reside en familia. La edad no define por sí misma a un grupo como problema en las sociedades actuales. Algunas personas ancianas se encuentran en estado de necesidad, muchas otras no lo están. Lo que ocurre a las personas más ancianas, en relación con las que han nacido después, es que ya estaban en desventaja desde antes, por haberse desarrollado en sociedades cuyas condiciones económicas, sanitarias, culturales y sociales eran peores. Ésta es una cuestión para destacar, porque pone de relieve la importancia de las condiciones sociales, entre las que se encuentran los tipos de políticas y servicios sociales, que configuran los distintos tipos de vejez existente entre sociedades, y dentro de una sociedad. La vejez no es un hecho sólo biológico, sino también cultural. Es una realidad que se construye socialmente (BAZO, 1992b).

En la actualidad dos son los problemas en la vejez en las sociedades desarrolladas y los dos grandes temas para las polí ticas: la persistencia de la pobreza a pesar de la mejoría experimentada, y los cuidados de salud de las personas enfermas crónicas y discapacitadas. Proveer y financiar los cuidados de larga duración de las personas ancianas son las cuestiones que más presión ejercen en la elaboración de las políticas, y los grandes retos a los que se enfrentan las políticas en el próximo siglo (BAzO, 1998; Rodríguez y SANCHO, 1995). Respecto al futuro es preciso analizar ciertos factores. La relación de la esperanza de vida, longevidad, y prevalencia y duración de las enfermedades crónicas definirán el nivel de dependencia entre las personas ancianas, lo que a su vez influirá en el sistema sanitario. Puede hipotetizarse sobre el impacto posible del control de las enfermedades y de las condiciones de vida en la esperanza de vida y la dependencia. Existe un consenso acerca de que la esperanza de vida no puede prolongarse mucho más, que habrá pocos cambios en este sentido.

El impacto en el sistema de salud del notable volumen previsto de personas ancianas será consecuencia de las condiciones de vida de la población y del alcance de la dependencia. No se disponen de buenas medidas del bienestar físico de las personas, del bienestar relativo de los diferentes grupos de edad en la población a lo largo del tiempo. Existe un consenso acerca de que las personas mayores se encuentran en mejor estado de salud y de apariencia física que como estaban hace cuarenta años. Habría también una mejora en la medida en que la proporción de personas que han alcanzado los setenta años ha aumentado. Podremos, pues, contar en un futuro con una población en mejores condiciones que lo esperado, y la dependencia que hubiera podido esperarse entre las personas mayores 
hace cuarenta años no sería de esperar en la actualidad. Las consecuencias de la mejora de las condiciones de la población anciana pueden influir en los cambios de las políticas. Con respecto a la Seguridad Social se espera que la edad de jubilación se retrase a los setenta años. Cuando a principios de siglo se establece la edad de jubilación a los sesenta y cinco años, pocas personas llegaban a jubiladas, y sólo una pequeña proporción vivía el tiempo suficiente para disfrutarla. Esto supone que los costos se están posponiendo. Si no se produce ningún otro cambio puede esperarse sencillamente que el volumen de cuidados de salud que se requería anteriormente se experimentará por las personas a una edad más tardía, pero las persones necesitarán los cuidados. Se observa la importancia para la política de salud de la investigación e información en los cambios en los niveles de salud en la estructura de edades cambiante. Interesa sobremanera investigar sobre el comienzo y duración de las enfermedades crónicas.

La utilización en el futuro de los cuidados de larga duración depende de las tendencias de los niveles de discapacidad de las personas ancianas, y todavía nadie sabe qué ocurrirá, cómo variarán los modelos de morbilidad. Las tres teorías que especulan al respecto señalan tres modelos distintos (GARBER, 1989: 257-259). Como las mejoras en la morbilidad han acompañado a las mejoras en la esperanza de vida, se espera que el promedio en morbilidad de las personas ancianas decaiga y que la discapacidad se produzca poco antes de la muerte (FRIES y FUCHS). La segunda concepción es que las personas que antes morían ahora sobreviven enfermas y discapacitadas, por lo que la dependencia puede aumentar con el tiempo (VerBRUGGE). La tercera perspectiva (Poterba y SUMmers) apunta a un mantenimiento de la situación actual debido a que el incremento de la vulnerabilidad en los ancianos será compensado con las mejoras en la morbilidad debido a la medicina y otros avances. La discapacidad de las personas ancianas supervivientes del futuro depende de las condiciones específicas que mejoran por el cuidado médico. Si existe una prevención o tratamiento efectivos para los problemas de salud de los futuros ancianos pueden estar menos discapacitados que los actuales (p. 258). Aumentar sólo la esperanza de vida puede llevar a aumentar el porcentaje de personas ancianas dependientes, debido a que las enfermedades crónicas y discapacidad son más habituales en la edad avanzada. Dado el incremento de las personas de más edad se espera que aumente el volumen de población anciana necesitada de cuidados de larga duración.

Debe tenerse en cuenta de cara al futuro que ciertas enfermedades son debidas a factores que pueden ser controlados por las personas para evitar riesgos graves para la salud, como la hipercolesterimia, hipertensión o tabaquismo, que conllevan riesgos graves de sufrir enfermedades cardiovasculares. En otras se desconocen las causas que las motivan y, por tanto, se carece de medidas de prevención, como es el caso de la enfermedad de Alzheimer, que es responsable de la mayor parte de los casos de demencia, lo que conduce a una necesidad de cuidados, sea en el hogar, sea en instituciones, que puede durar varios años. El aumento de los costos de los cuidados de salud está causando gran preocupación social y se convierte en un elemento de confrontación política. Se analizan los costes altos producidos en la atención de las personas con enfermedades crónicas y moribundas, en las secciones de agudos donde se utiliza tecnología que es muy costosa. Se proponen cambios en el cuidado de los moribundos para que pasen del sistema de salud al de servicios sociales. A la vez se debaten los costes causados por la atención a las personas crónicas en una institución o en el entorno comunitario. Se asume que mientras las familias acepten realizar ese cuidado resultará más barato. La cuestión del análisis de los costos puede variar si todos los servicios que son prestados en la comunidad deben ser pagados. Las propuestas de cambio se centran en los mecanismos de financiación, la fuente de pago o la fuente de los servicios. Una idea subyace y es que las personas ancianas deben recibir el mejor cuidado posible aunque no resulte clara la definición del desideratum. Suele entenderse por el mejor cuidado posible proveer servicios intensivos para prolongar la vida lo máximo posible.

Varios son los factores que influyen en el alza de los costes aparte del incremento de la inflación en determinados momentos, como son el aumento de la población que recibe cuidados, el incremento vertiginoso de los costes del cuidado hospitalario, y el creciente uso de técnicas de diagnóstico y tratamiento muy caras, debido a la alta tecnología utilizada. Otro factor es de carácter demográfico, es decir, existe un aumento de las personas de más edad, que sufren más enfermedades que los grupos más jóvenes, van más al médico, son hospitalizadas con mayor frecuencia y son más largas sus estancias hospitalarias. Todo ello repercute en un gasto en salud varias veces mayor para este grupo que para otros grupos más jóvenes. El gasto entre las personas mayores no es homogéneo, sino que es sólo una pequeña proporción de entre ellas la que efectúa una gran utilización de los servicios y, por tanto, la que realiza el mayor gasto. El coste más alto se produce en el último año de sus vidas debido al uso intensivo de los servicios conforme se acerca el momento de la muerte (Neugarten, 1994: 159; Montgo. MERY Y BORGATTA, 1987: 244).

Desde la Economía de la Salud se entiende que no es el factor envejecimiento el único que influye en el alza de los costes de la salud, ni siquiera el más decisivo. El problema que se plantea en la actualidad en las sociedades desarrolladas no es el de las enfermedades agudas, sino el de las enfermedades crónicas, pero el sistema médico se basa todavía más en curar que en cuidar. Las personas que sufren enfermedades crónicas, enfermedades de larga duración, son atendidas principalmente en la familia, de manera que la gestión de la enfer- 
medad se desarrolla más en el hogar que en el hospital, y son enfermos/as que necesitan servicios sanitarios y sociales (NEU. GARTEN, 1994). Al aumentar el número de personas muy ancianas ha aumentado la necesidad de cuidados de larga duración, pero también han aumentado para otros grupos de edad: niños, jóvenes y adultos. Eran personas que en otras épocas morian y que ahora siguen viviendo, requiriendo atenciones y cuidados. La calidad de vida para todas las personas es un valor y una meta en las sociedades más desarrolladas, lo que podríamos entenderlo como un indicador de «civilización». Las políticas sociosanitarias que se diseñen y los servicios que se provean, su diversidad y extensión, serán indicadores también de la solidaridad social en una sociedad y de su nivel de civilización.

Todo lo visto conduce a analizar el tema de los valores acerca de la conservación de la vida. Sostener un tipo u otro de valores conduce a elaborar políticas diferentes. Se habla del derecho a morir y surgen asociaciones que defienden tal derecho, por considerar que es una restricción de la propia libertad no permitir esa elección a la persona cuando es a costa de utilizar medios extraordinarios para conservar la vida, o por medio de lo que también se denomina «encarnizamiento terapéutico». Por otro lado, los valores diferentes acerca de la conservación de la vida conducen a impedir a algunas personas el acceso a ciertos recursos, como es el caso en algunos países de la diálisis para las personas ancianas, a las que se priva de ello. Queda implícita la idea de que puede vivir aquella persona que puede devolver a la sociedad parte de lo que ha recibido, o aquella que puede pagarse privadamente el servicio. Surge la pregunta de cuáles son los estándares bajo los que pueden ser negados los servicios en el área de la salud. Si el sistema público se rige únicamente desde esos principios de rentabilidad social, el acceso a la salud puede quedar restringido sólo a los ancianos/as que puedan costearla. Se quebraría profundamente el principio básico del Estado de Bienestar.

Si se aceptan las definiciones de «muerte cerebral», la cuestión ya no es si los recursos sanitarios se utilizan de forma productiva para la sociedad, sino si lo que se está tratando es de conservar tejido vivo. Ese asunto es de importancia cuando se constata el incremento del gasto en cuidados de salud en los enfermos terminales, o incluso el coste del cuidado de las personas enfermas de SIDA, para las que por el momento no existe curación. Además, en ciertos casos surge la pregunta de si merece la pena vivir en ciertas condiciones, como es el caso de algunas personas discapacitadas que desean morir. Desde la perspectiva de la calidad de vida, se considera que es una crueldad forzar a las personas a vivir en contra de su voluntad. Las cosas se complican si por debilidad física o mental de la persona se implican otras personas, como ocurre en algunas parejas. Los hospicios en el mundo anglosajón es una alternativa para facilitar la fase terminal de las personas enfermas. En el momento actual en todos los países las presiones acerca de los costos ponen sobre el tapete el tema de los valores. Se produce un debate social y se influye en la definición de las políticas.

Un componente fundamental de los cuidados de larga duración es el cuidado informal, que son los servicios prestados por la familia y amigos. Los costes y cantidad de esos servicios que no pasan por el mercado no se han medido adecuadamente aunque existe evidencia de que son amplios (GARBER, 1989: 261). En España, como en otras sociedades, la gran mayoría de las personas ancianas que reciben cuidados de salud son atendidas por su familia, y son mujeres las que asumen el rol de cuidadoras. Se entiende que en las dos o tres últimas décadas en el plano internacional se está produciendo un «descubrimiento de los cuidadores» aunque hayan existido siempre (Hunter y MACPHERSON, 1993: 67). Las primeras investigaciones sobre cuidado informal comenzaron en los años cincuenta (UNGERSON, 1987; TeNNESTEDT y MCKInLAY, 1989). Se da una coincidencia entre la consideración de los servicios en la comunidad entendidos más «humanitarios» que los institucionales, y la valoración económica de unos y otros, vistos los primeros más baratos. Sin embargo, esto está siendo cuestionado. Las políticas para las personas ancianas se están centrando en el mantenimiento y potenciación de los cuidados informales, de la familia concretamente, y comienza a verse los servicios sociales públicos como sustitutivos de la familia. Se considera que los grupos prioritarios que necesitan atención son precisamente las personas que experimentan algún tipo de necesidad, pero que viven solas y aisladas socialmente.

En España recientemente se investiga sobre el cuidado informal. Siendo pocas todavía las investigaciones en España sobre el tema, sin embargo, en las escasas publicaciones (BAzO, 1998; García SANZ, 1997; Bazo y Domínguez, 1996a, 1996b; INSERSO, 1995b: 267; RodRíGuez, 1994) parece apreciarse los costos económicos que suponen la pérdida de empleo, o reducción de jornada de las personas cuidadoras. Otros costos son el deterioro de la salud, alteraciones de carácter, conflictos con la pareja, sentimientos de culpabilidad por desatender a otros miembros de la familia, como son los hijos pequeños o el esposo, de restricción de libertad, y otros problemas, que manifiestan sobre todo las mujeres, que son las tres cuartas partes de las personas cuidadoras. En España las mujeres cuidadoras actuales son principalmente amas de casa. Se estima que el $4,7 \%$ de las personas de dieciocho y más años prestan ayuda informal a personas ancianas, lo que supone que alrededor de un millón y medio de personas ejercen de cuidadoras (INSERSO, 1995b: 284). Los importantes cambios demográficos, económicos, culturales y sociales acaecidos, transforman las sociedades desarrolladas. Todo ello tiene consecuencias en la familia que experimenta cambios notables en sus formas, estructura y tamaño, así como en sus funciones tradicionales. 
El potencial de cuidados de una sociedad se acostumbra a calcularlo teniendo en cuenta a las mujeres - no a las personas- de cuarenta y cinco a sesenta y nueve años, con relación a la población de setenta y más años. Ese potencial en España ha pasado de 2,5 mujeres de esa edad por persona anciana en 1960, a 1,5 en 1990, lo que supone una reducción del $62 \%$ (WALKER, GUILlEmard y AlBER, 1993: 54). Además del factor de cambio demográfico que influye en esa disminución del potencial de cuidados, la progresiva incorporación de las mujeres al mundo del trabajo, su cada vez mayor preparación académica con niveles más altos de instrucción que sus predecesoras, junto con las presiones económicas que hacen cada vez más necesario un segundo salario en la familia, hace prever que la situación actual cambiará en el próximo futuro. El cuidado comunitario - como alternativa al institucional — apareció como la panacea que resolvería los problemas que surgen de la necesidad de hacer frente a los cuidados de larga duración en las personas ancianas. Ocurre a veces, y es una realidad en España, que esos cuidados se realizan por la familia (INSERSO 1995) y sin apoyo alguno de los servicios sanitarios ni sociales (Bazo 1998; Bazo y Domínguez, 1996a, 1996b). Se considera (WILKIN y HugES, 1991: 173) que el concepto de comunidad provee a los políticos de un medio para reconciliar su necesidad de hacer frente a los problemas organizacionales de las personas ancianas, con su deseo de adoptar una perspectiva humanista. Lo que se entiende por comunidad no tiene un significado unívoco. En muchas de sus concepciones, sin embargo, se entiende idealmente que la comunidad satisface determinadas necesidades de las personas. Puede ocurrir que las personas ancianas que residen en sus antiguas casas en sus antiguos barrios se vean rodeadas de personas que ya no conocen. Han muerto algunas que conocían de siempre, o se han trasladado a otro mejor, entre ellas grupos de personas nacidas allí que son más jóvenes. Pero se han instalado grupos diversos de personas: emigrantes, estudiantes, o jóvenes que comienzan su vida independiente en alojamientos y barrios que son más baratos.

Es más fácil adscribirse a la idea de comunidad que identificarla. Lo que se asume en general es que es lo opuesto a la institucionalización (HUGMAN, 1994: 135). Se asocia así al cuidado en casa y tiende a identificarse en las políticas con el mantenimiento en el domicilio. Un problema suele ser la concentración de los servicios comunitarios en las áreas metropolitanas, en detrimento de las rurales, lo que produce desigualdades geográficas. Otros se refieren a la adecuación de los servicios a las necesidades de la población anciana, y quién define los criterios para precisar las necesidades, al margen de la dificultad de la definición del propio concepto de necesidad. En Europa se observan orientaciones distintas para las políticas que van desde el intento de sustituir la atención comunitaria por los servicios informales, pasando por quienes pretenden completar y apoyar los servicios informales, hasta quienes pro- veen a las personas ancianas de los cuidados comunitarios necesarios sin complemento de los informales (JAMIESON e ILLSEY, 1993). En Europa, en distintos países se conceden remuneraciones a las personas que prestan servicios asistenciales informales tanto a las personas ancianas o enfermas como a los hijos/as cuidadores/as (EvERS y LEICHSENRING, 1994). Otra cuestión a analizar es si las políticas sociales deben tener en cuenta las diferencias entre los distintos modelos de relación familiar, las divisiones por género en el cuidado familiar y las diferencias estructurales entre familias según la clase social.

La idea del cuidado comunitario aparece ligada al principio de que el mejor lugar para las personas mayores es su propia casa, con la ayuda de los servicios comunitarios. Sin embargo, lo que se observa es un fallo de los servicios sanitarios y sociales que no proveen a la persona anciana ni a sus familiares del apoyo suficiente para poder hablar de un cuidado compartido entre la persona anciana necesitada, la familia y el Estado o los servicios públicos. En la actualidad en España como en otras sociedades, a pesar del discurso sobre la conveniencia de la atención en la comunidad, la mayor parte del presupuesto de los servicios sociales para personas ancianas se lo llevan los servicios residenciales. La escasez de servicios de atención domiciliaria y otros como centros de día y hospitales de día, tienen como consecuencia el uso abusivo de los servicios provistos por las mujeres, de manera que son ellas y las familias las que continúan soportando los costos sociales de la dependencia. La ideología del familismo refuerza más la desigualdad entre los miembros de la familia y constriñe la demanda de los servicios públicos (QURESHI y WALKER, 1991).

Las políticas y los servicios sociales se elaboran teniendo en cuenta una concepción determinada de la familia y la comunidad. La familia se ha entendido compartiendo responsabilidades entre sus miembros y ofreciéndose mutuamente contraprestaciones, como algo propio de las «obligaciones familiares». Todavía hoy la palabra obligación se escucha de las personas que cuidan familiares enfermos crónicos. En una investigación de carácter cualitativo realizada por la autora en las Comunidades del País Vasco, Cataluña y Madrid (BAzo, 1998; BAzo y Dominguez, 1996a, 1996b) se observa -como en otros estudios- la construcción social del rol de cuidadora en las mujeres. Ellas, como el resto de la sociedad, asumen como natural que deben ser las mujeres las que cuiden de las personas enfermas de la familia, de cualquier edad. Se basa en la asunción del rol de ama de casa como el principal, aunque se ejerzan además otros, que tienen que ver con la realización de otras actividades, incluida la del trabajo remunerado fuera del hogar, que representaría un rol secundario, al contrario que para los varones. El rol de cuidadoras de las mujeres es visto como un rol que toman de por vida como la promesa del matrimonio «hasta que la muerte nos separe» (PEACE, 1991). El cuidado de las personas ancianas, y entre ellas las mujeres son 
mayoría, sigue corriendo principalmente por cuenta de las mujeres de la familia. Pero cuando se trata de los servicios formales, también son fundamentalmente mujeres las que los prestan, lo que demuestra la estrecha asociación entre las mujeres y su rol como cuidadoras. Cuando los varones son cuidadores principales, como suele ocurrir entre las personas casadas, la cantidad de cuidados que proporcionan son similares a los de las mujeres (TENNESTEDT y MCKINLAY, 1989).

La concepción de la familia se basa en una estructura en la que el varón es el proveedor de los ingresos y la mujer la proveedora de los cuidados y el servicio. La diferenciación de roles en la familia lleva a las mujeres a especializarse como amas de casa y madres. Se considera que su tiempo empleado en el desempeño de tales roles es libre en términos de disponibilidad y costo (Allan, 1990: 17). Cuando se crea la Seguridad Social, aunque se tiene en cuenta que algunas mujeres casadas participan en el mercado de trabajo, la idea que predomina es la del varón que gana el sustento, y mantiene al resto de los miembros dependientes de la familia (esposa e hijos, principalmente). Se considera que la creación de los Estados de Bienestar se basó en el trabajo no pagado de las mujeres en el hogar, y en el pagado pero con salarios bajos de las mujeres en las instituciones de bienestar. Del mismo modo, la estructura de prestaciones reflejaba el status dependiente de las mujeres y los deberes domésticos como su prioridad, mientras se entendía que la responsabilidad primordial de los varones se encontraba en el mercado de trabajo. Sigue sucediendo que las políticas sociales actuales que ponen el énfasis en el cuidado comunitario tienden a reforzar la ideología del familismo al asumir que la familia es el lugar idóneo para el cuidado de las personas ancianas enfermas, y que las mujeres son los familiares más apropiados para asumir esa tarea (WALKER, 1991).

Por otro lado, los servicios sociales comunitarios tienden a ser prestados no en función del grado de necesidad de las personas, sino del apoyo familiar de que disponen, sobre todo si se tiene una hija cerca (PEACE, 1991; QuRESHI y WaLKER,
1989). Los límites entre el cuidado formal e informal para los servicios sociosanitarios son rígidos (HÆL y BRAMLEY, 1992: 122). Existe, en general, un escaso seguimiento y desconexión entre ambos. Sucede que no se han desarrollado suficientemente unos servicios de apoyo a las personas cuidadoras. Puede darse al mismo tiempo que haya personas que soportan una carga exageradamente desproporcionada de cuidado, y otras personas en necesidad que están desatendidas por el entorno familiar y comunitario. Se observa que faltan mecanismos para evaluar el nivel de carga que es aceptable y que protejan los derechos de las personas cuidadoras. Una preocupación para el trabajo social público es prevenir el fallo de la asistencia familiar. Los trabajadores sociales del sector público se ocupan sólo de las situaciones en las que los sistemas privados de atención no funcionan o funcionan mal.

Aunque en algunas Comunidades Autónomas como Navarra, Euskadi o Cataluña los servicios sociales pueden tener un desarrollo algo más amplio que en otras, lo cierto es que en España la importancia de la familia como cuidadora es abrumadoramente superior a la que puede tener en otras sociedades con sistemas de protección social más extensa e intensivamente desarrollados. Se observa desde una perspectiva internacional que existe una relación inversamente proporcional entre la utilización de las fuentes de cuidado formal e informal. A mayor desarrollo de las funciones protectoras del Estado, menor (y distinta) aportación familiar, y viceversa. Sin embargo, en todas las sociedades la familia sigue siendo fuente fundamental de cuidados (TENNESTEDT y McKinlay, 1989; JamiESON e IlLSLEY, 1993; MONTGOMERY y HaTCH et al., 1987).

Los retos a las políticas y los servicios sanitarios y sociales en sociedades cada vez más envejecidas —donde se han producido transformaciones notables en la tecnología, la producción económica, la familia, la posición de las mujeres y el mundo de los valores - presentan debates y discusiones que requieren de una participación social.

* Catedrática de Sociología, Facultad de Ciencias Económicas y Empresariales, Universidad del País Vasco. 


\section{Bibliografía}

ALLAN, Graham (1990), Family Life, Oxford: Blackwell

Bazo, María Teresa (1999a), Las personas mayores en Europa, Madrid: Ediciones de Politica Exterior

Bnzo, María Teresa (ed.) (1999b), Envejecimiento y sociedad: Una perspectiva internacional, Madrid: Editorial Médica Panamericana.

- (1998), «El cuidado familiar en las personas ancianas con enfermedades cró. nicas: el caso de los pacientes con enfermedad de Alzheimern, Revista Española de Geriatria y Gerontologia, 33 (1): 49-56.

- (1994), «Abuelos y nietos: La familia como centro privilegiado de intercambio entre gencraciones», pp. 269.373, en Premio Bancaixa de Investigación 1993, Valen. cia: Bancaixa.

- (1992a), La ancianidad del futuro, Barcelona: SG Editores

- (1992b), «La vejez como construcción cultural», Revista de Gerontologia, 4: 237-242.

- (1990), La sociedad anciana, Madrid: Centro de Investigaciones Sociológicas y Siglo XXI.

Bnzo, Maria Teresa, y Domínguez-Alcón, Carmen (1996a), «Los cuidados familiares de salud en las personas ancianas, y las políticas sociales», Revista Española de Investigaciones Sociológicas, 73: 43-56.

- (1996b), «Los cuidados familiares de salud para las personas ancianas», Quaderns: 23.27

Béland, François, y Zunzunegui, M. Victoria (1995), «El perfil de las incapacidades funcionales de las personas mayores», Revista de Gerontologia, 5, pp. 232-244.

CIRES (1993), La realidad social en España 1991-1992, cap. 10 Tercera edad Sabadell: Puresa: 579.667

Collado, A.; Dominguez-Alcón, C., y De Micuel, J. M. (1991), Las estructuras de la prevención de deficiencias: El caso de Barcelona, Madrid: Ministerio de Asuntos Socinles.

CRUz, Pcpa, y Coвo, Rosa (1990), Situación social de los viejos en España, Madrid: Centro de Investigaciones Sociológicas, Estudios y Encuestas.

EVERS y LEICHSENRING (1994), «Asistencia informal remunerada: Una cuestión de creciente importancia», Revista de Gerontologia, 4, No. 2: 114-124.

Dízz Nicolss, Juan (1996), Los mayores en la Comunidad de Madrid, Madrid: Fundación Caja de Madrid.

Fiernindez-Mnyornlas, Gloria, y Rodríguez Rodricuez, Vicente (1995), «La capacidad funcional de los ancianos españoles», Revista de Gerontologia, vol. 5 , No. 1: 16-12.

Garber, Alan M. (1989), «Long-term care, wealth, and health of the disabled elderlyn, pp. 257-277, en David A. WISE (ed.), The Economics of Aging, Chicago: The National Bureau of Economic Research.

Garcin Sanz, Benjamín (ed.) (1997), Envejecimiento en el mundo rural: Problemas y soluciones, Madrid: Ministerio de Trabajo y Asuntos Sociales.

GarCh Sanz, Benjamin (1996), La sociedad rural ante el siglo xxI, Madrid: Ministerio de Agricultura, Pesca y Alimentación.

- «Demografía de la vejez» (1999), en María Teresa Bazo (ed.), Envejecimiento y sociedad: Una perspectiva intermacional, Madrid: Editorial Médica Panamericana.

Hill, Michael, y Bramley, Glen (1992), Analysing Social Policy, Oxford: Blackwell

Hugman, Richard (1994), Ageing and the Care of Older People in Europe, Londres: The MacMillan Press.
HUNTER, David, y MACPHERSON, Isobel (1993), «Influencia de los cuidadores informales sobre la provisión de servicios y las decisiones de asignación», pp. 67.92, en JAImIESON, Anne, y ILLSLEY, Raymond, Comparación de políticas europeas de atención a las personas ancianas, Barcelona: SG.

INSERSO (1995a), Las personas mayores en España. Perfiles. Reciprocidad familiar, Madrid: Ministerio de Asuntos Sociales.

- (1995b), Cuidados en la vejez: El apoyo informal, Madrid: Ministerio de Asuntos Sociales.

Jamieson, Anne, y ILlsley, Raymond, (1993), Comparación de politicas europeas de atención a las personas ancianas, Barcelona: SG

Miguel, Jesús M. de, et al. (1994), Capítulo 6 «Salud y sanidad», pp. 823-1106, en Quinto informe sociológico sobre la situación social en España: La salud para todos en el año 2000, Madrid: Fundación Foessa, 2 vols.

MONTGOMERY, Rhonda J. V., y BorgotTA, Edgar F. (1987), en BorgotTA, Edgar F. y Montgomery, Rhonda J. V. (eds.), Critical Issues in Aging Policy: Linking Research and Values, Londres: Sage Publications.

MontGomery, Rhonda J. V., Hatch, Laurie R, et al. (1987), «Dependency, family extension, and long-term care policym, en BORGOTTA, Edgar F., y MONTGOMERY, Rhonda J. V. (eds.), Critical Lssues in Aging Policy: Linking Research and Values, Londres: Sage Publications, pp. 161-177.

Neugarten, Bernice, y Neugarten, Dail A. (1994), «Policy issues in an aging society», en Martha Storandt y Gary R. VAndenbos, The Adult Years: Continuity and Change, Washington: American Psychological Association: 143-167.

Neugarten, Bernice, L. (1974), «Age groups in American society and the rise of the young old", Annals of the American Academy of Political Science, 515 187-198.

PEACE, Sheila (1991), «The forgotten female: Social policy and older women», pp. 61-86, en Phlulpson, Chris, y Walker, Alan (eds.), Ageing and Social Policy: A Critical Assessment, Aldershot: Gower.

QURESHI, Hazel, y WALKER, Alan (1991), «Caring for elderly people: the family and the state», pp. 109-127, en Chris PHLLLPSON y Alan WaLKER, Ageing and Social Policy: A Criticas Assessment, Aldershost: Grower, pp. 109-127.

QuReshi, Hazel, y WaLker, Alan (1989), The Caring Relationship: Elderly People and their Families, Londres: MacMillan Education.

Revista Española de Geriatria y Gerontologia (1995), 30 (3). Monográfico sobre Politica social y atención gerontológica a las personas mayores dependientes.

Rodríguez, Josep A. (1994), Envejecimiento y familia, Madrid: Centro de Investigaciones Sociológicas y Siglo XXI.

Rodriguez Rodriguez, Pilar, y Sancho, M.a Teresa (1995), «Nuevos retos de la política social de atención a las personas mayores: Las situaciones de fragilidad», Revista Española de Geriatria y Gerontologia, 30 (3): 141.152.

SalvaGe, Ann V. (1995), Who Will Care?, Shankill, Co. Dublin: Loughlinstown House.

TENNSTEDT, Sharon L., y McKINLAY, Jhon B. (1989), «Informal care for frail older persons", pp. 145.166, en OrY, Marcia G., y Bond, Katheleen (eds.), Aging and Health Care: Social Science and Policy Perspectives, Londres: Routledge.

Ungerson, Clare (1987), Policy is Personal: Sex, Gender and Informal Care, Londres: Tavistock Publications.

VALLÉs, Miguel, y CeA D'AnCONA, M. A. (1994), «Los mayores», pp. 821.908, en Amando DE MIGUEL, La sociedad española 1993-1994: Informe sociológico de la Universidad Complutense, Madrid: Alianza

WALKER, Alan (1995), «The family and the mixed economy of care, can the be integrated?», pp. 201-220, en ALLEN, Isobel, y PERKINS, Elizabeth (eds.), The Future of Family Care for Older People, Londres: HMSO. 
- (1991), «The relationship between the family and the state in the care of older people», Canadian Joumal on Aging / La Revue Canadienne du Vieillissement, vol. 10, No. 2: 94-112.

WalkeR, Alan;-Gulllemard, Anne-Marie, y AlBer, Jens (1993), Older People in Europe: Social and Economic Policies, Bruselas: Comision de las Comunidades Europeas.
Walker, Alan, y Maltby, Tony (1997), Ageing Europe, Buckingham: Open University Press.

WLKIN, David, y HUGES, Beverly (1991), "The elderly and the health services», en Chris PhuLIPSON y Alan WaLKer, Ageing and Social Policy: A Critical Assessment, Aldershost: Grower: 163-183. 